\title{
Optical Frequency Link between Torino and Firenze for remote comparison between $\mathrm{Yb}$ and $\mathrm{Sr}$ optical clocks
}

\author{
Davide Calonico $^{1}$, Cecilia Clivati ${ }^{1,2}$, Giovanni A. Costanzo ${ }^{2}$, Aldo Godone ${ }^{1}$, Filippo Levi ${ }^{1}$, Marco Marchetti ${ }^{3}$, \\ Alberto Mura ${ }^{1}$, Marco Prevedelli ${ }^{4}$, Marco Schioppo ${ }^{3}$, Guglielmo M. Tino ${ }^{3}$, Massimo E. Zucco ${ }^{1}$, Nicola Poli ${ }^{3}$ \\ ${ }^{1}$ Istituto Nazionale di Ricerca Metrologica, Torino, Italy, \\ ${ }^{2}$ Politecnico di Torino, Torino, Italy \\ ${ }^{3}$ Dipartimento di Fisica e Astronomia and LENS, Università di Firenze, INFN Firenze, Italy, \\ ${ }^{4}$ Dipartimento di Fisica, Università di Bologna, Bologna, Italy \\ Email: d.calonico@inrim.it, nicola.poli@unifi.it
}

\begin{abstract}
The Italian Institute of Metrology (INRIM), the European Laboratory for NonLinear Spectroscopy (LENS), the Physics and Astronomy Department of the University of Firenze (UNIFI) and the Politecnico of Torino started a project to establish a $650 \mathrm{~km}$ optical fiber link that will enable remote frequency comparisons between high accuracy microwave and optical clocks of INRIM and UNIFI-LENS and an absolute measurement of the UNIFI-LENS Sr clock versus INRIM Cesium fountains.

We report the overall architecture of the project, the present status of the experiment and the preliminary tests performed on the equipments.
\end{abstract}

\section{INTRODUCTION}

Nowadays, atomic Cs fountains offer an accuracy of few parts in $10^{16}$ [1], while optical frequency standards demonstrated the possibility of an accuracy even beyond [2], [3]. Presently the most used transfer techniques to compare atomic frequency standards and to disseminate their accuracy and stability rely on satellite techniques, that limit the performances of fountains and even more of optical standards [4]. In fact, satellite techniques give an uncertainty contribution of few parts in $10^{16}$ only after at least 20 days of continuous measurements, whilst atomic fountains tipically reach this value in half a day and optical frequency standards in $100 \mathrm{~s}$ or less. Aside from transportable optical standards, optical fiber links seem the only way to compare remote atomic clocks without a limitation of their possibilities, over continental scale for the moment.

In recent years, the reliability and the performances of optical links have been demonstrated over hauls of several hundreds kilometres, allowing frequency dissemination and remote frequency comparisons with a resolution better than $10^{-19}$ over few thousands seconds [5], [6], [7]. Presently, several European National Metrology Institutes and research institutes are working to set up the branches of a continental optical network.

Different techniques have been used to obtain frequency dissemination through optical fiber. For example, RF amplitude modulation of an optical carrier has been exploited
[8], whereas the direct transfer of an optical carrier, with lower losses and higher resolution, is preferred nowadays. Also, the direct transfer of an optical comb is under study [9], even if at the moment this tecnhique needs a more complex infrastructure, not always available on commercial networks. To obtain a stable transmission of the optical carrier over long distances, the transmitted radiation has to be frequency stabilized, but also, the phase noise due to temperature variations, vibrations and mechanical noise in the fibers has to be compensated.

INRIM in Torino and UNIFI-LENS in Firenze, in collaboation with Politecnico of Torino and University of Bologna, have started a project to set up an optical fiber link between them. Both institutes are involved in high accuracy atomic clocks experiments: INRIM operates two primary Cs atomic fountains and is developing an optical clock based on laser cooled neutral Ytterbium, whereas at UNIFI-LENS a $\mathrm{Sr}$ optical clock is under development and high resolution spectroscopy measurements are being perfomed. The optical link will allow firstly an absolute characterization of the UNIFI-LENS Sr clock with respect to the SI second, that is realized at INRIM through the two Cs fountains; at the same time, a direct comparison between the $\mathrm{Yb}$ and $\mathrm{Sr}$ optical clocks will be possible, allowing also remote tests of fundamental physics. The experiment will give a contribution to the research on novel techniques for time and frequency transfer through optical fibers, and also to the establishment of a pan-European network of optically-linked laboratories.

The collaboration with Politecnico of Torino and University of Bologna will be beneficial to the development of the optoelectronic equipment to implement the optical link.

\section{A $650 \mathrm{~km}$ OPTICAL LINK IN ITALY}

The overall scheme of the optical link is shown in Figure 1. A commercial optical fiber connects INRIM (hereafter, local end) and UNIFI-LENS (remote end) laboratories, and an ultrastable laser radiation in the optical C-band (1542 nm 


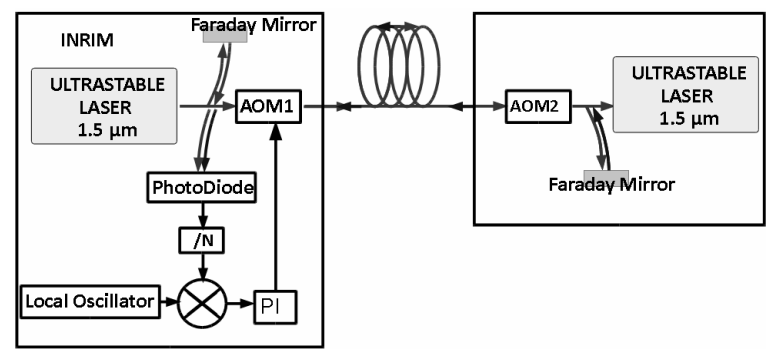

Fig. 1. Conceptual scheme for phase noise cancellation of the optical fiber link. The beatnote between the injected and round-trip radiation is revealed on a fast photodiode, frequency divided $(/ \mathrm{N})$ and compared to a stable reference in order to generate an error signal. This signal is then integrated and sent to the acousto-optical modulator AOM1, that acts as actuator for frequency correction. At the remote end, AOM2 shifts the optical carrier in order to distinguish the round-trip signal from spurious reflections along the fiber path.

in the present experiment) is sent from INRIM to UNIFILENS. In each laboratory, the laser frequency is compared using optical femtosecond combs to the frequency of an atomic frequency standard. The laser source could be either a fiber laser or an External Cavity Diode Laser (ECDL) [10] and it is frequency stabilized on a Fabry-Pérot cavity in vacuum, made of ULE glass, thermally stabilized and suspended in a configuration with low sensitivity to vibrations. The frequency stability of the present experimental set-up could reach an Allan deviation of few parts in $10^{15}$, flicker frequency noise limited.

To implement the noise Doppler cancellation technique, at the remote end in Firenze part of the radiation will be reflected back to INRIM and compared with the input signal to actively detect and compensate the phase noise contribution of the optical fiber. An Acousto-Optic Modulator (AOM1) is placed at the local end of the link and used as an actuator for frequency correction. A second modulator at the remote end (AOM2) shifts the frequency of the back-reflected radiation, to distinguish spurious reflections from the round-trip signal.

The optical fiber infrastructure is managed by the Italian National Research and Educational Network (NREN), named GARR. For the first two years of the experiment, the link between INRIM and UNIFI-LENS will have at disposal a couple of dedicated fibers. Then, a Dense Wavelength Division Multiplexing (DWDM) architecture will be implemented, with a dedicated $100 \mathrm{GHz}$ wide channel (the channel 44 of the International Telecommunication Union grid, ITU44), suitable for transferring radiation at $1542 \mathrm{~nm}$.

This optical fiber structure will also be used for the implementation of GARR-X, the Italian new generation fiber network for data traffic dedicated to research acivities, upgrading the present architecture that is still not full-optical. The overall link length is $650 \mathrm{~km}$, estimated loss is $194 \mathrm{~dB}(0.3 \mathrm{~dB} / \mathrm{km})$. This evaluation is provided by GARR on the basis of direct measurements and of design models. Losses compensation will be achieved by 9 optical amplification stations along the fiber haul: bidirectional Erbium Doped Fiber Amplifiers

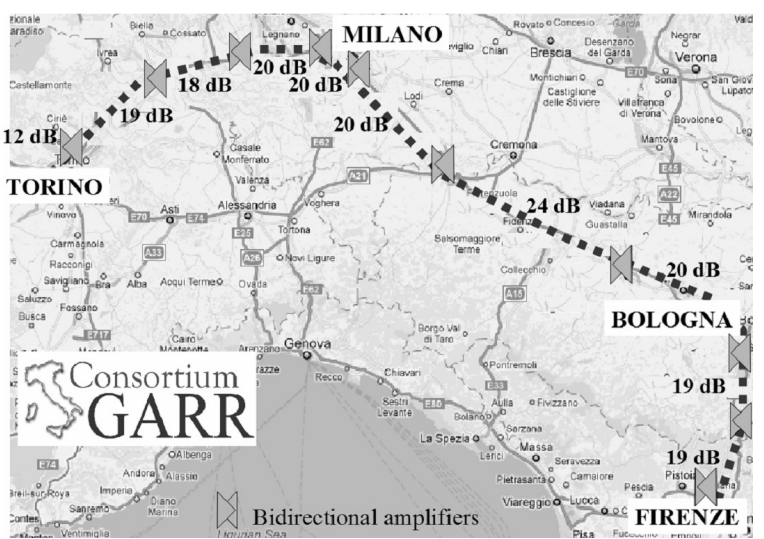

Fig. 2. The architecture of the Italian $650 \mathrm{~km}$ link under development.

(EDFA) will be used, while at UNIFI-LENS the signal will be regenerated using a local ECDL. The gain of each EDFA will be remotely controlled by GSM drivers, and should be kept as low as $15-20 \mathrm{~dB}$ to prevent lasing effects. Therefore we need to place an amplifier each $20 \mathrm{~dB}$ losses to avoid degradation in the Signal/Noise ratio.

Figure 2 shows the effective fiber path; we report the housing possibilities for the amplification stations, together with the attenuation for each span. The housing locations are still under consideration, and a certain degree of freedom is offered to the experiment. In particular, we are working to split the two spans with $30 \mathrm{~dB}$ losses to reduce them to $20 \mathrm{~dB}$, with one more amplification station.

\section{Clock DEVElopments IN THE TWO LABORATORIES}

The experiment will compare the $\mathrm{Sr}$ optical clock in Firenze with the atomic clock ensemble in Torino. INRIM currently operates two laser-cooled Cs fountains, ITCsF1 and ITCsF2. Since 2003 ITCsF1 has been realizing the SI second at $5 \times 10^{-16}$ relative accuracy level, and has contributed for more than 25 times to the generation of the Internatinal Atomic Timescale (TAI) of the Bureau International of Poids and Mesures (BIPM). ITCsF2 is a cryogenic fountain designed with the target of $1 \times 10^{-16}$ relative accuracy level. Both exhibit a frequency instability of about $2 \times 10^{-13}$ at $1 \mathrm{~s}$ averaging time in terms of Allan deviation [11], [12].

A neutral $\mathrm{Yb}$ optical clock is under development. Presently, Ytterbium atoms are prepared in a double stage Magneto Optical Trap (MOT), exciting the dipole transition at $399 \mathrm{~nm}$ and the intercombination line at $556 \mathrm{~nm}$. The first MOT is loaded by a thermal beam pre-slowed by a counterpropagating laser radiation at $399 \mathrm{~nm}(5 \mathrm{~mW})$; the MOT laser intensity is about $2 \mathrm{~mW} / \mathrm{cm}^{2}$ for each beam, while the static magnetic field is $45 \mathrm{G} / \mathrm{cm}$. Up to $5 \times 10^{5}$ atoms are loaded in the first MOT and then 50\% are trasferred into the second stage MOT, where they are cooled down to temperature of tens of $\mu \mathrm{K}$. Currently, we are implementing the off resonance dipole trap at $759 \mathrm{~nm}$ for the Lamb-Dicke regime spectroscopy. The $578 \mathrm{~nm}$ clock laser has been realized [13]. Figure 3 shows the stability of the clock laser, reaching a flicker frequency 




Fig. 3. Allan deviation of the Yb clock laser without (upper curve) and with (lower curve) seismic damping.

flor of $3 \times 10^{-15}$ limited by the noise contribution of transfer optical fibers that we are currently going to compensate.

A Ti:Sa optical comb will be used to compare the Yb clock laser at $578 \mathrm{~nm}$ with the Cs fountains, whereas a fiber optical comb is going to be used for the optical link.

At UNIFI-LENS, a ${ }^{88} \mathrm{Sr}$ optical clock is in advanced development. Its absolute characterization will be performed by the optical link respect to the SI definition of the second realized at INRIM.

Presently, the double stage MOT at $461 \mathrm{~nm}$ (blue MOT) and at $689 \mathrm{~nm}$ (red MOT) and a 1D lattice trap at $813 \mathrm{~nm}$ have been realized, and prelinary spectroscopic measurements are being carried on.

About $50 \%$ of the atoms are transferred from the red MOT into the lattice trap resulting in about $5 \times 10^{5}{ }^{88} \mathrm{Sr}$ atoms. The observed lifetime of the trap is about $1.4 \mathrm{~s}$ indicating low heating effects due to amplitude or frequency noise coming from the un-stabilized $813 \mathrm{~nm}$ source.

The clock laser at $698 \mathrm{~nm}$ is generated by a diode laser locked on a ULE Fabry-Pérot ultrastable cavity and has an estimated frequency stability of $10^{-15}$ for averaging times between $10-100 \mathrm{~s}$ [14].

Applying a magnetic field of $1.1 \mathrm{mT}$ and using an interaction time of $\mathrm{T}=300 \mathrm{~ms}$, the clock transition has been observed with a linewidth of $410 \mathrm{~Hz}$ [15], as shown in Figure 4. For these measurements we applied a constant magnetic field $B$ (along the polarization of the clock laser field and the trapping field) by inverting the current on one of the MOT coils, whereas laser intensity $I$ is estimated from the laser waist $w_{0}$ on the atoms and the beam power.

It is possible to find the transition on a day to day basis even without a precise calibration of the laser frequency by adding a $200 \mathrm{kHz}$ chirping on the clock laser frequency (with $2 \mathrm{~s}$ period) and by increasing the interaction time on the atomic cloud to $1 \mathrm{~s}$.

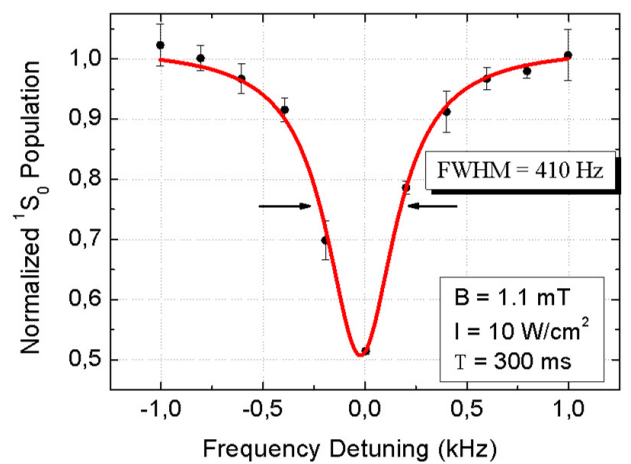

Fig. 4. Magnetic Field induced spectroscopy on the clock transition for ${ }^{88} \mathrm{Sr}$ isotope.

\section{OPTICAL LINK EXPERIMENTAL SET-UP}

At INRIM, the laser source at $1542 \mathrm{~nm}$ to be transmitted in the link is already operative and characterized, as well as the phase noise compensation system.

The source is a commercial diode laser, frequency locked to a ULE high finesse (120000) cavity through a Pound-DreverHall technique. We evaluated its performances by a beatnote between two identical systems. Residual instability is $10^{-14}$ at $1 \mathrm{~s}$, limited by acoustic noise and vibrations on the reference cavity. An upgrade of the seismic damping system is ongoing, and from phase noise spectrum evidence we expect to be able to achieve the same results of the Yb clock laser, as the reference system is basically identical.

The phase noise arising in the fiber is compensated by a phase locked loop (PLL) that acts on a AOM. First, a real link was simulated using $100 \mathrm{~km}$ of fiber spool in the laboratory and the performances of our noise cancellation loop were evaluated measuring the beatnote between the injected laser and the radiation at the remote end of the link.

Then, the system was demonstrated on a real fiber loop that is part of the urban internet network and has both ends in the laboratory. The link has a DWDM architecture and uses a dedicated (ITU44) channel $100 \mathrm{GHz}$ wide. Total lenght is $47.6 \mathrm{~km}$ and losses are $\sim 20 \mathrm{~dB}$. Figure 5 shows the noise spectrum of the free running and compensated urban link. The system cancels the fiber noise down to the limitation imposed by the delay, as evaluated in [16].

The interferometer is protected against temperature variations and acoustic noise. Nevertheless, the link stability over long averaging times is limited by residual temperature and pressure variations over the short out-of-loop fibers. Figure 6 reports the fractional instability of the free running and compensated $100 \mathrm{~km}$ spooled fiber, acquired with a $\Lambda$-type counter; this does not correspond to the Allan deviation in presence of white phase noise. Further discussion and scaling formulas are discussed in [17]. 


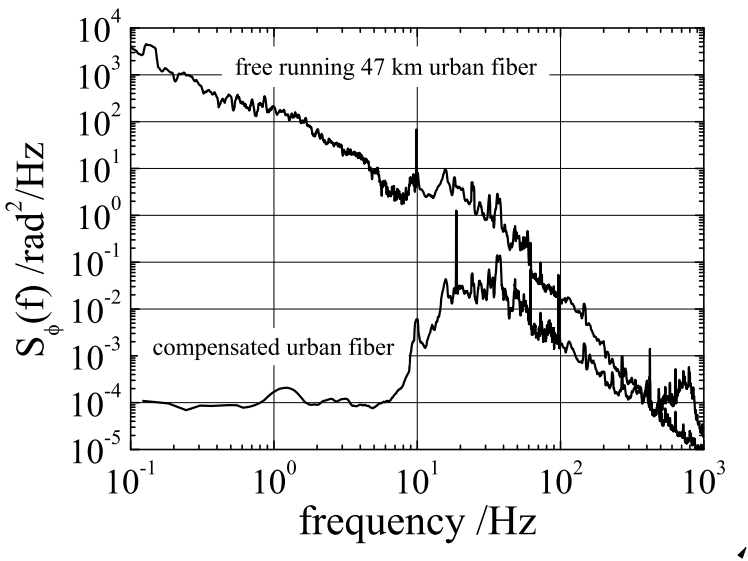

Fig. 5. Phase noise spectra of the free running (upper curve) and compensated (lower curve) urban link.

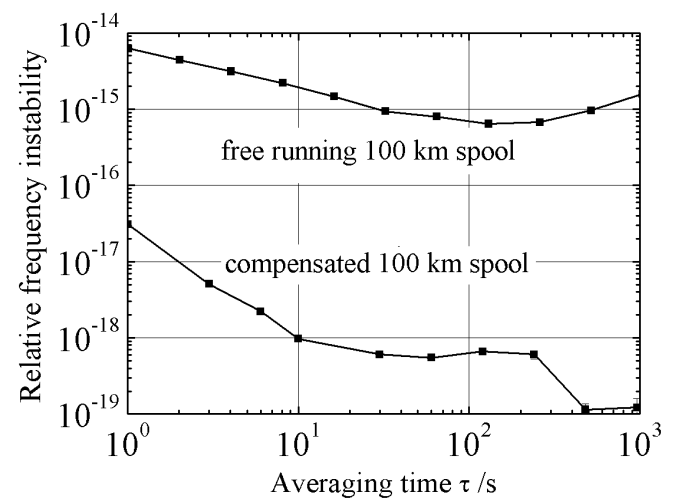

Fig. 6. Relative instability of the free running and compensated $100 \mathrm{~km}$ spool, acquired with a $\Lambda$-type counter.

\section{CONClusion}

We presented the ongoing work to set up a DWDM optical link of about $650 \mathrm{~km}$ that will connect the Italian National Metrological Institute INRIM to the laboratories of UNIFILENS. This link will allow remote frequency comparisons between the atomic clocks of the two Institutes and a characterization of the UNIFI-LENS ${ }^{88} \mathrm{Sr}$ clock with respect to the SI second. Moreover, the Torino-Firenze optical link will be the backbone of an Italian Network for optical fiber link dissemination of highly stable and accurate time and frequency signals. This network could be integrated in a wider system of optical links spreading all over Europe, allowing to realize a unique metrological platform composed of the many Cs fountains and optical clocks now present in Europe.

\section{ACKNOWLEDGMENT}

The authors would like to acknowledge funding by Compagnia di San Paolo for INRIM link equipments, and Cassa di Risparmio di Firenze for UNIFI-LENS link equipments.

\section{REFERENCES}

[1] T. Parker, "Long-term comparison of caesium fountain primary frequency standards", Metrologia vol. 47, pp 1-10, 2010.
[2] C. W. Chou, D. B. Hume, J. C. J. Koelemeij, D. J. Wineland, and T. Rosenband, "Frequency Comparison of Two High-Accuracy $\mathrm{Al}^{+}$Optical Clocks, Phys. Rev. Lett. vol. 104, pp. 070802-4, Febr. 2010.

[3] N. Huntemann, M. Okhapkin, B. Lipphardt, S. Weyers, C. Tamm, and E. Peik, "High-accuracy optical clock based on the octupole transition in ${ }^{171} \mathrm{Yb}^{+}$, Phys. Rev. Lett. vol. 108, pp. 090801-5, Mar. 2012.

[4] A. Bauch, J. Achkar, S. Bize, D. Calonico, R. Dach, R. Hlavac, L. Lorini, T. Parker, G. Petit, D. Piester, K. Szymaniec, P. Uhrich, "Comparison between frequency standards in Europe and the USA at the $10^{-15}$ uncertainty level", Metrologia, vol. 43, pp. 109-120, Febr. 2006.

[5] A. D. Ludlow, T. Zelevinsky, G. K. Campbell, S. Blatt, M. M. Boyd, M. H. G. de Miranda, M. J. Martin, J. W. Thomsen, S. M. Foreman, J. Ye, T. M. Fortier, J. E. Stalnaker, S. A. Diddams, Y. Le Coq, Z. W. Barber, N. Poli, N. D. Lemke, K. M. Beck, C. W. Oates, "Sr Lattice Clock at $1 \times 10^{-16}$ Fractional Uncertainty by Remote Optical Evaluation with a Ca Clock", Science vol. 319, no. 5871, pp. 1805-1808, Febr. 2008.

[6] K. Predehl, G. Grosche, S. M. F. Raupach, S. Droste, O. Terra, J. Alnis, Th. Legero, T. W. Hänsch, Th. Udem, R. Holzwarth, H. Schnatz, "A 920-Kilometer Optical Fiber Link for Frequency Metrology at the 19th Decimal Place", Science vol. 336, no. 6080, pp. 441-444, Apr. 2012.

[7] O. Lopez, A. Haboucha, F. Kéfélian, H. Jiang, B. Chanteau, V. Roncin, C. Chardonnet, A. Amy-Klein, G. Santarelli, "Cascaded multiplexed optical link on a telecommunication network for frequency dissemination", Opt. Expr. vol. 18, no. 16, pp. 16849-16857, Aug. 2010.

[8] O. Lopez, A. Amy-Klein, M. Lours, C. Chardonnet, G. Santarelli, "Highresolution microwave frequency dissemination on an $86-\mathrm{km}$ urban optical link", Appl. Phys. B vol. 98, no. 4, pp. 723-727, 2010.

[9] G. Marra, R. Slavík, H. S. Margolis, S. N. Lea, P. Petropoulos, D. J. Richardson, P. Gill , "High-resolution microwave frequency transfer over an 86-km-long optical fiber network using a mode-locked laser", Opt. Lett. vol. 36, no.4, pp. 511-513, 2011.

[10] C. Clivati, D. Calonico, C. E. Calosso, G. A. Costanzo, F. Levi, A. Mura, A. Godone, "Planar-Waveguide External Cavity Laser Stabilization for an Optical Link with $10^{-19}$ Frequency Stability", IEEE Trans. Ultrason. Ferroelectr. Freq. Contr. vol. 58, no. 12, pp. 2582-2587, Dec 2011.

[11] F. Levi, C. Calosso, D. Calonico, L. Lorini, E. K. Bertacco, A. Godone, G. A. Costanzo, B. Mongino, S. R. Jefferts, T. P. Heavner, E. A. Donley, "Cryogenic fountain development at NIST and INRIM: preliminary characterization", IEEE Trans. Ultrason. Ferroelectr. Freq. Contr. vol. 57, no. 3, pp. 600-605, Mar. 2010.

[12] F. Levi, D. Calonico, L. Lorini, A. Godone, "IEN-CsF1 primary frequency standard at INRIM: accuracy evaluation and TAI calibrations", Metrologia vol. 43, no. 6, pp. 545, 2006.

[13] M. Pizzocaro, G. A. Costanzo, A. Godone, F. Levi, A. Mura, M. Zoppi, D. Calonico, "Realization of an Ultrastable 578-nm Laser for an $\mathrm{Yb}$ Lattice Clock", IEEE Trans. Ultrason. Ferroelectr. Freq. Contr. vol. 59, no. 3, pp. 426431, Mar. 2012.

[14] M. G. Tarallo, N. Poli, M. Schioppo and G. M. Tino. "A high stability semiconductor laser system for a ${ }^{88} \mathrm{Sr}$-based optical lattice clock", Appl. Phys B vol. 103, no. 1, pp. 17-25, 2011.

[15] N. Poli, M. G. Tarallo, M. Schioppo, C. W. Oates and G. M. Tino. "A simplified optical lattice clock", Appl. Phys. B vol. 97, pp. 27-33, 2009.

[16] P. A. Williams, W. C. Swann, N. R. Newbury, "High stability transfer of an optical frequency over long fiber-optics links", J. Opt. Soc. Am. B, vol. 25, no. 8, pp. 1284-1293, Jul. 2008.

[17] S. T. Dawkins et al, "Considerations on the measurementof the stability of oscillators with frequency counters", IEEE Trans. Ultrason. Ferroelectr. Freq. Control, vol. 54, no. 5, pp. 918-925, May 2007. 